\title{
REFERENCE
}

\section{Transport Coefficients and Molecular Dynamics: Alternatives}

Mountain, R. D.

U. S. DEPARTMENT OF COMMERCE

Technology Administration

National Institute of Standards

and Technoiogy

Physical and Chemical Properties Division

Gaithersburg, MD 20899-8380

\section{QC \\ 100 \\ .456 \\ \# 7170 \\ 2004}

\section{NLT}

National Instifute of Standards and Technology

Technology Administration

U.S. Department of Commerce 



\section{Transport Coefficients and Molecular Dynamics: Alternatives}

Mountain, R. D.

U. S. DEPARTMENT OF COMMERCE

Technoiogy Administration

National Institute of Standards and Technology

Physical and Chemical Properties Division

Gaithersburg, MD 20899-8380

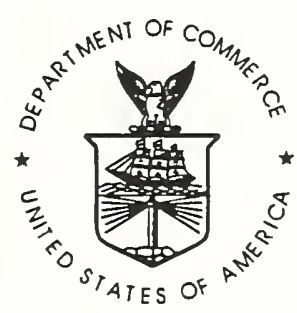

U.S. DEPARTMENT OF COMMERCE Donald L. Evans, Secretary

TECHNOLOGY ADMINISTRATION

Phillip J. Bond, Under Secretary for Technology

NATIONAL INSTITUTE OF STANDARDS

AND TECHNOLOGY

Arden L. Bement, Jr., Director 


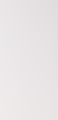




\title{
Transport coefficients and molecular dynamics: Alternatives
}

\author{
Raymond D. Mountain \\ Physical and Chemical Properties Division \\ Chemical Science and Technology Laboratory \\ National Institute of Standards and Technology \\ Gaithersburg, MD 20899-8380
}

\section{Abstract}

The task of using molecular dynamics to determine the shear viscosity and the thermal conductivity of a model fluid is examined in detail for the Lennard-Jones fluid. The equilibrium time correlation function approach is compared with three nonequilibrium methods, Poiseuille Flow, Momentum Impulse Relaxation, and Reversed Perturbation Nonequilibrium Molecular Dynamics. The conventional wisdom is that the equilibrium approach requires very long simulation times in order to obtain statistically significant results. This study finds that only the Reversed Perturbation Nonequilibrium Molecular Dynamics method has the possibility of significantly reducing the simulation time required to obtain "good" estimates for the thermal conductivity. All of the reliable methods examined can produce accurate estimates for the shear viscosity with shorter simulation times than are needed for the determination of the thermal conductivity.

\section{Introduction}

In this note, we consider some schemes for estimating transport coefficients of fluids that do not make use of the Green-Kubo formulation of transport coefficients [1]. The GreenKubo methods are derived from statistical mechanics and yield correct values when properly evaluated. However, they require very long run times to obtain reliable estimates. In the alternative methods discussed below, the system is arranged so that the properties of the system can be mapped onto a calculable hydrodynamic flow and the transport coefficient(s) are then obtained as parameters that make the computations match the hydrodynamic solution, just as is done experimentally. The objective of this study is to identify computationally efficient methods for estimating transport coefficients for fluids that are defined in terms of molecular structure and interactions. The methods examined here are labeled Poiseuille Flow, Momentum Impulse Relaxation, and Reversed Perturbation Nonequilibrium Molecular Dynamics. In all of the simulations discussed in this note, the equations of motion were integrated using the Beeman algorithm [2]. A neighbor table was used to improve the efficiency of the simulations [3] with the result that the simulation time approximately scales as the number of particles. 
In order to compare results for the methods to be discussed, we list in Table 1 some Green-Kubo based estimates of the transport coefficients thermal conductivity $\lambda$ and shear viscosity $\eta$ of the Lennard-Jones fluid for several dense fluid state points [4]. As usual, all properties are listed in terms of the Lennard-Jones potential parameters for energy $(\epsilon)$ and length $(\sigma)$ and in terms of the mass $m$ of the Lennard-Jones particle and are therefore dimensionless. The Lennard-Jones potential, $\phi(r)$, has the form

$$
\phi(r)=4 \epsilon\left[(\sigma / r)^{12}-(\sigma / r)^{6}\right] .
$$

The time unit $\tau$ for simulations is

$$
\tau=\sqrt{m \sigma^{2} / \epsilon}
$$

The tests of the methods reported here are all for the Lennard-Jones fluid and the values in Table 1 are used to evaluate the effectiveness of the alternative methods. Other sets of transport coefficients for the Lennard-Jones fluid determined using the Green-Kubo method are found in the literature $[5,6,7,8,9]$. This is not an all-inclusive list of sources. While the Lennard-Jones fluid is an idealization, it has been shown that when the potential parameters are appropriately chosen, the model predicts values for the shear viscosity and thermal conductivity for the rare gases argon, krypton, and xenon that are in good agreement with experimental values [10].

Table 1. A set of Green-Kubo based thermal conductivity $\lambda$ and shear viscosity $\eta$ transport coefficients for the Lennard-Jones fluid at specified number densities $\mathrm{n}$ and temperatures $\mathrm{T}$ are listed here. The estimated uncertainty in the transport coefficients is reported to be on the order of $5 \%$ [4].

\begin{tabular}{|c|c|c|c|}
\hline $\mathrm{n}$ & $\mathrm{T}$ & $\lambda$ & $\eta$ \\
\hline 0.85 & 0.745 & 7.66 & 3.28 \\
0.85 & 0.908 & 7.18 & 2.64 \\
0.85 & 1.033 & 7.34 & 2.75 \\
0.85 & 1.318 & 8.03 & 2.56 \\
0.85 & 1.503 & 8.19 & 2.44 \\
0.85 & 1.976 & 8.08 & 2.28 \\
0.85 & 2.905 & 8.46 & 2.31 \\
0.75 & 1.008 & 4.72 & 1.66 \\
0.75 & 1.330 & 5.64 & 1.68 \\
0.75 & 2.017 & 6.06 & 1.51 \\
0.75 & 2.913 & 7.02 & 1.53 \\
0.65 & 1.588 & 4.14 & 0.88 \\
0.65 & 1.851 & 4.46 & 0.99 \\
0.65 & 3.564 & 5.30 & 1.00 \\
\hline
\end{tabular}


The three nonequilibrium methods are discussed in the next three sections. In each case, the method is first reviewed based on the existing literature. Then the way we have implemented the method is described and some results for the Lennard-Jones fluid are presented. The final section contains a discussion of the merits of the methods and some recommendations on which methods are potentially useful for the simulation of more complex fluids.

In order to make explicit comparisons of the computational effort involved in the different methods, we first introduce the equilibrium time correlation function approach known as the Green-Kubo method and present results for the first state point listed in Table 1.

The Green-Kubo method expresses transport coefficients as time integrals of equilibrium time correlation functions of fluctuating currents $[11,12]$. For the shear viscosity, the current is the off-diagonal element of the stress tensor $\mathcal{P}_{\alpha \beta}$,

$$
\mathcal{P}_{\alpha \beta}=\sum_{i=1}^{N} m_{i} v_{i}^{\alpha} v_{i}^{\beta}+\sum_{j>i} r_{i j}^{\alpha} F_{i j}^{\beta}
$$

The shear viscosity is

$$
\eta=\frac{1}{V k_{B} T} \int_{0}^{\infty}<\mathcal{P}_{\alpha \beta}(0) \mathcal{P}_{\alpha \beta}(t)>d t
$$

where $\langle\ldots\rangle$ indicates an ensemble average of the enclosed quantity. The corresponding current for the thermal conductivity, the heat current $\mathcal{J}_{\alpha}$, takes the form in the microcanonical ensemble [12],

$$
\mathcal{J}_{\alpha}=\sum_{i=1}^{N}\left[v_{i}^{\alpha}\left[\frac{1}{2} m v_{i}^{2}+\sum_{j \neq i} \phi\left(r_{i j}\right)\right]+\frac{1}{2} \sum_{j \neq i}\left(\vec{F}_{i j} \cdot \vec{v}_{i}\right) r_{i}^{\alpha} \cdot\right]
$$

The thermal conductivity is

$$
\lambda=\frac{1}{V k_{B} T^{2}} \int_{0}^{\infty}<\mathcal{J}_{\alpha}(t) \mathcal{J}_{\alpha}(t)>d t .
$$

It is important to realize that the heat current expression is ensemble dependent [12].

The shear viscosity and thermal conductivity have been determined for a 500 LennardJones particle system in the microcanonical ensemble. That is for constant number of particles $\mathrm{N}$, constant system volume $\mathrm{V}$, and constant energy $\mathrm{E}$. The total momentum of the system is set to zero. A total of $10^{6}$ time steps of duration $0.01 \tau$ were needed to obtain satisfactory convergence of the current-current time correlations to zero for long times. This amounts to an overall time interval of $10^{4} \tau$. A total of 3985 time origins separated by $2.5 \tau$ were generated so that the time correlation functions are averaged over 
3985 independent samples. The influence of system size on the transport coefficients is less than the uncertainty in the estimates for $\mathrm{N} \geq 500[13,14,15,16]$. In addition to the explicit results for the state with $\mathrm{T}=0.76$ and $\mathrm{n}=0.85$ that are presented below, the other states listed in Table 1 have been run in order to estimate the uncertainties in the transport coefficients. A rough estimate of the uncertainty in these coefficients can be obtained by determining the variance of the values of the integrals of the three time correlation functions [17]. In general the uncertainties in the shear viscosity are on the order of $5 \%$ and those in the thermal conductivity are on the order of $10 \%$.

\section{Shear viscosity}

The time correlation functions were generated for the $x y, x z$, and $y z$ components of the stress tensor and are shown in Fig. 1.1 as $\mathcal{P}^{\alpha \beta}(t)$ scaled so that

$$
\eta=\int_{0}^{\infty} \mathcal{P}^{\alpha \beta}(t) d t
$$

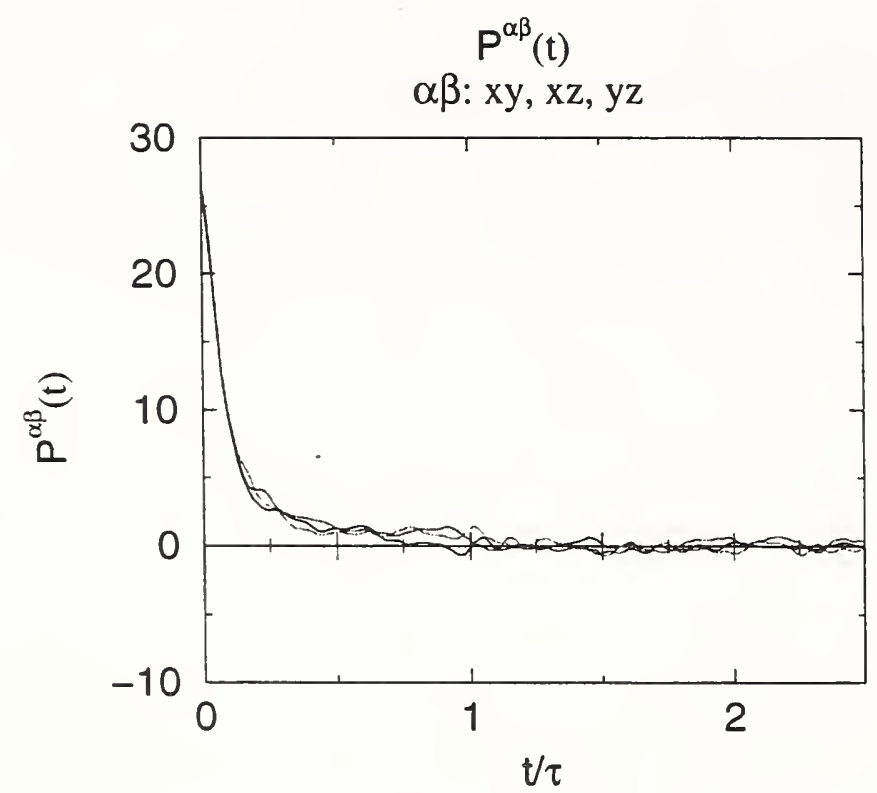

Fig. 1.1. The normalized time correlation functions that appear in eq. 1.5 are shown as functions of time.

Fig. 1.2 shows the average of the three time correlation functions and the integral of the averaged correlation function. The "long time" value of the integral is 3.38 , in reasonable agreement with the value listed in Table 1 . The standard uncertainty for this state is \pm 0.2 . 


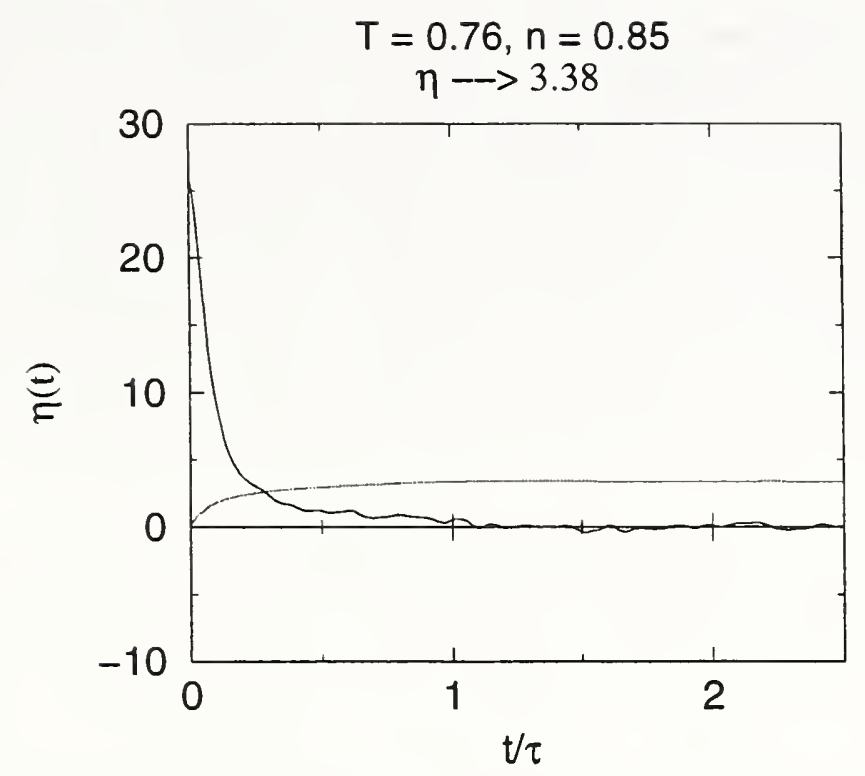

Fig. 1.2. The averaged time correlation functions (solid line) and the time integral for the shear viscosity (dotted line) are shown here.

\section{Thermal conductivity}

The current-current correlation functions, $J_{\alpha}(t)$, scaled so that

$$
\lambda=\int_{0}^{\infty} J_{\alpha}(t) d t
$$

are shown in Fig. 1.3 for the $\mathrm{x}, \mathrm{y}$, and $\mathrm{z}$ components of the heat current.

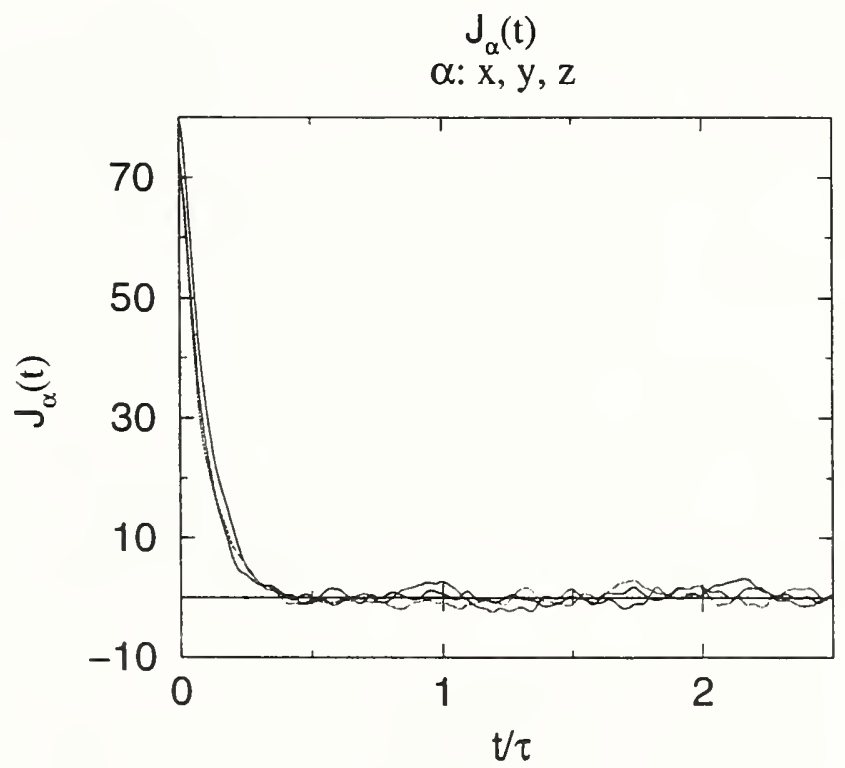

Fig. 1.3. The averaged time correlation functions for the heat current are shown here.

Fig. 1.4 shows the average of the three time correlation functions and the integral of the 


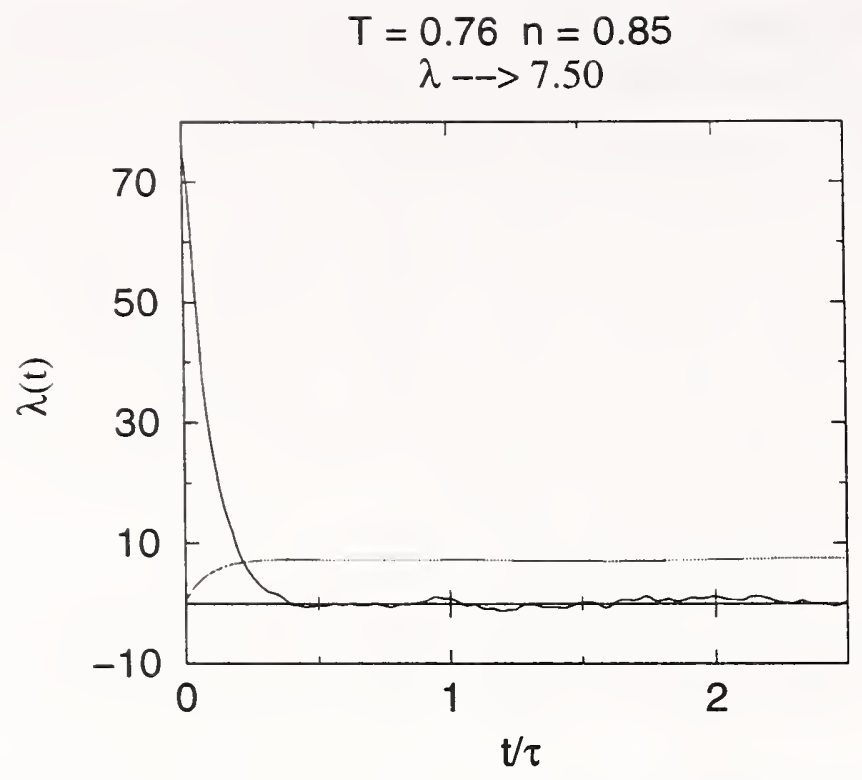

Fig. 1.4. The averaged time correlation functions (solid line) and the time integral for the thermal conductivity (dotted line) are shown here.

averaged correlation function. The "long time" value of the integral is 7.50 , in reasonable agreement with the value listed in Table 1 . The standard uncertainty in this value is \pm 0.8 .

Note that the long time limit for the time correlation functions for this state point is reached when $t \leq 1.5 \tau$ so that the individual samples of the correlation functions are independent.

\section{Poiseuille Flow}

Poiseuille flow refers to the flow of a fluid, subject to a uniform body force, between parallel plates with stick boundary conditions. If the plates are located at $\dot{y}= \pm h$ and the flow is in the $x$ direction, then according to linear Navier-Stokes hydrodynamics, the steady state $\mathrm{x}$-component of the flow velocity, $u_{x}(y)$, satisfies

$$
u_{x}(y)=-\frac{n F_{e}}{\eta}\left[y^{2}-h^{2}\right]
$$

where $F_{e}$ is the driving force for the flow (such as gravity), $n$ is the number density, and $\eta$ is the shear viscosity. The corresponding temperature profile in the slit is

$$
T(y)=T_{0}-\frac{\left(n F_{e}\right)^{2} y^{4}}{(12 \eta \lambda)}
$$

where $T_{0}$ is the midchannel temperature and $\lambda$ is the thermal conductivity of the fluid. The heat generated by the viscous damping is dissipated by thermal conduction through the walls of the slit [18]. 
This suggests that it might be feasible to determine both the thermal conductivity and the shear viscosity of a fluid from a single nonequilibrium molecular dynamics simulation of Poiseuille flow. This would entail determining both the temperature profile and the flow velocity profile. Denis Evans and co-workers have examined the issues associated with the molecular dynamics simulation of Poiseuille flow in a series of papers [19, 20, 18, 21, 22, 23]. Their objective was not the extraction of transport coefficients but rather understanding the issues involved in performing steady-state nonequilibrium simulations. Some of the salient results from this body of work are discussed in the following paragraphs.

Their simulations used a simple arrangement that is sketched in Fig. 2.1. The simulation cell of dimensions $L_{x} \times L_{y} \times L_{z}$ is periodic in all three dimensions. The plates are represented by three layers of atoms at one boundary (dark circles in the figure) that are tethered to fcc sites by a harmonic potential. Note that only one set of layers is in the basic simulation cell. The other layer is just the periodic image of the first layer. The wall atoms also interact with the fluid molecules by a specified potential. The wall atoms are thermostated so that the heat generated in the fluid is removed from the system. The fluid atoms are not thermostated and, in addition to intermolecular interactions, are subject to a uniform, time independent driving force in the $x$ direction. The use of a uniform external driving force means that density gradients that would be produced by a pressure gradient are absent. Once a steady state is achieved, the velocity profile, $u_{x}(y)$ and the temperature profile in the fluid are determined as time averages.

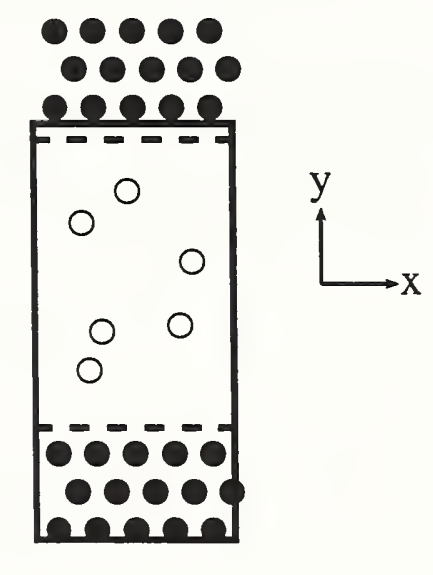

Fig. 2.1. A sketch of the simulation scheme used by Todd and Evans [18]. The dark frame indicates the simulation cell (the z-direction is normal to the page) and the dashed lines indicate the effective size of the channel $(2 \mathrm{~h})$.

The initial studies used a short range WCA-type potential to describe the interactions 
between the fluid atoms and between the fluid atoms and the wall atoms. For this simple system, it was necessary to postulate the existence of a strain rate term in the heat flux vector in order to accurately describe the temperature profiles generated in the simulations. In addition to the fourth power term in the Navier-Stokes solution, this coupling generates a quadratic term. When a molecular fluid (liquid chlorine) was examined, the quadratic term in the temperature profile was not observed [23]. Also, when ethane and hexane in fairly narrow channels were simulated, the quartic form described the temperature profiles except near the walls.

A major concern in many of these studies was how to accurately and efficiently generate the temperature profile. The kinetic approach uses the particle velocity minus the flow velocity to determine the local temperature, namely

$$
k_{B} T(y)=\frac{1}{3 N(y)} \sum_{i=1}^{N} m_{i}\left(\mathbf{v}_{i}(y)-\mathbf{u}(y)\right)^{2}
$$

where the sum is over the molecules at a distance $y$ from the center of the channel. Some special sampling methods were developed to facilitate evaluation of spatial distributions without recourse to the "bins" used in histogram methods [20]. The kinetic approach is computationally inefficient for nonequilibrium simulations as it requires two simulations, one to determine the flow profile $\mathbf{u}(\mathrm{y})$ and then a second simulation to determine $T(y)$ or, if the distribution is generated within a single simulation by taking ratios of the velocity at a position divided by the density at that position. It requires long runs to reduce the statistical scatter.

An alternative method for determining the temperature that depends only on the configuration of the atoms was examined in some detail. The theory for this approach is described extensively in Ref. [24] and is based on the thermodynamic relation

$$
\frac{1}{k_{B} T}=\left(\frac{\partial S}{\partial E}\right)_{V}
$$

The details are not pertinent here, so we only consider the form that is readily implemented in a simulation without the need for multiple simulations or extra force loops. The result is

$$
\frac{1}{k_{B} T}=\left\langle-\sum_{i} \nabla_{i} \cdot \mathbf{F}_{i}\right\rangle /\left\langle\sum_{i} \mathbf{F}_{i}^{2}\right\rangle
$$

Another concern with the simulation of flow in a channel is how the width of the channel is reflected in departures from the predictions of the continuum Navier-Stokes equations [21]. Significant departures from the simple hydrodynamics predictions are found when the width of the channel $(2 \mathrm{~h})$ is less than about 10 particle diameters. The "stick" boundary 
condition does not strictly apply when the wall and fluid atoms are different in that the fluid-fluid and fluid-wall interactions are significantly different. The lesson appears to be that behavior near the walls shows significant departures from the predictions of NavierStokes hydrodynamics.

Finally, the examples in the literature are for short-range potentials. It is not known what the result would be if longer range interactions or Coulomb interactions were used. System size effects would be of particular concern. Coulomb interactions would have additional complications since periodicity in the $y$-direction would seem to be unphysical. One possible approach, that would not be too expensive computationally, would be to use the Ewald scheme for slab geometry that has a very large repeat distance in the $y$-direction with most of that region empty [25]. Alternatively, one could use the scheme proposed by Lekner $[26,27]$ although it is computationally quite expensive.

\section{Implementation}

In this section, we describe the implemention of the Poiseuille Flow simulation and also discuss the strengths and weaknesses of the proposed methods based on our experience. First we discuss a method that follows the approach of Evans, et al. Then some alternative methods are discussed. The examples are for atomic fluids interacting via a Lennard-Jones 12-6 potential. Quantities are reported in units of the Lennard-Jones parameters $\epsilon$ and $\sigma$ of the fluid atoms and the mass of the particles is the unit of mass of the fluid atoms.

In the first method to be considered, we followed the approach described above with one modification. Instead of placing a wall layer at one end of the simulation cell and considering the second wall layer to be the periodic image of the first layer, we considered having two explicit walls within the simulation cell, as indicated in Fig. 2.2.

Initially, we did not have periodic boundary conditions in the $y$-direction. However, this was not fully satisfactory as the wall layers tended to be pushed out by the fluid. After some experimentation, we settled on having the wall layers as indicated in Fig. 2 but with periodic boundary conditions in all three dimensions. With periodic boundary conditions, the two wall layers are in fact one larger layer with two surfaces in contact with the fluid. This eliminates most of the distortion of the walls by the fluid.

The code written to simulate Poiseuille flow contains 2000 Lennard-Jones fluid particles and 480 Lennard-Jones wall particles. The tethering positions for the wall molecules are specified to be fcc sites that are consistent with the $x$ - and $z$-dimensions of the simulation cell. The L-J $\sigma$ for the fluid-wall interaction is set to 1.2 to strongly inhibit penetration of the wall by the fluid and the $\sigma$ for the wall-wall interactions is set to $2^{-1 / 6}$ so that the "equilibrium" separation matches the tethering sites. The remainder of the Lennard-Jones parameters are set to unity for all interactions. The mass of all particles is unity. 


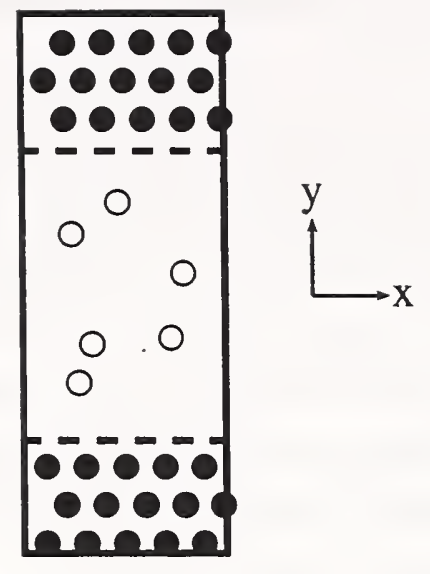

Fig. 2.2. A sketch of the simulation scheme used here. The dark frame indicates the simulation cell (the z-direction is normal to the page) and the dashed lines indicate the effective size of the channel (2h). Note that the upper wall layers are within the simulation cell, unlike Fig. 2.1.

The temperature of the wall particles is maintained by a Nosé-Hoover thermostat [28]. The equations of motion are integrated using the Beeman algorithm [2] with a time step of $0.01 \tau$. Initially, the system was equilibrated with no external driving force for a temperature of 1.0. The density, $n$ of the fluid in the region away from the walls settled out to be 0.825 .

A series of runs with driving forces $F_{e}$ varying from 0.01 to 0.05 were implemented. For this system, 0.01 was about the smallest driving force that resulted in a detectable temperature profile. The kinetic temperature is determined at each time step using the local flow velocity (eq. 2.3) at that instant. Once the driving force was applied, experience showed that a run of $2000 \tau\left(10^{5}\right.$ time steps) was needed to obtain a stationary temperature and flow velocity profile. Then a final run of $1000 \tau$ was made to obtain the profiles that were fit to the hydrodynamic forms. The profile information is collected in bins of width $1 \sigma$. An example of the profiles is displayed in Fig. 2.3.

The analysis procedes in two steps. First, the flow velocity of the fluid away from the wall is fit to a parabola using linear least squares,

$$
u_{x}\left(y_{i}\right)=u_{0}+u_{2}\left(y_{i}+y_{c}\right)^{2}
$$

where $y_{c}$, the "center" of the fluid layer is the position of the maximum in the parabola and $y_{i}$ is the position of bin $i$ relative to $y_{c}$. The centers the bins in the simulation cell are $Y=y_{i}+y_{c}$. An example of the fit is shown in Fig. 2.4.

This simplifies the next step of fitting the temperature profile $T\left(y_{i}\right)$ to

$$
T\left(y_{i}\right)=T_{0}-T_{4} y_{i}^{4}
$$




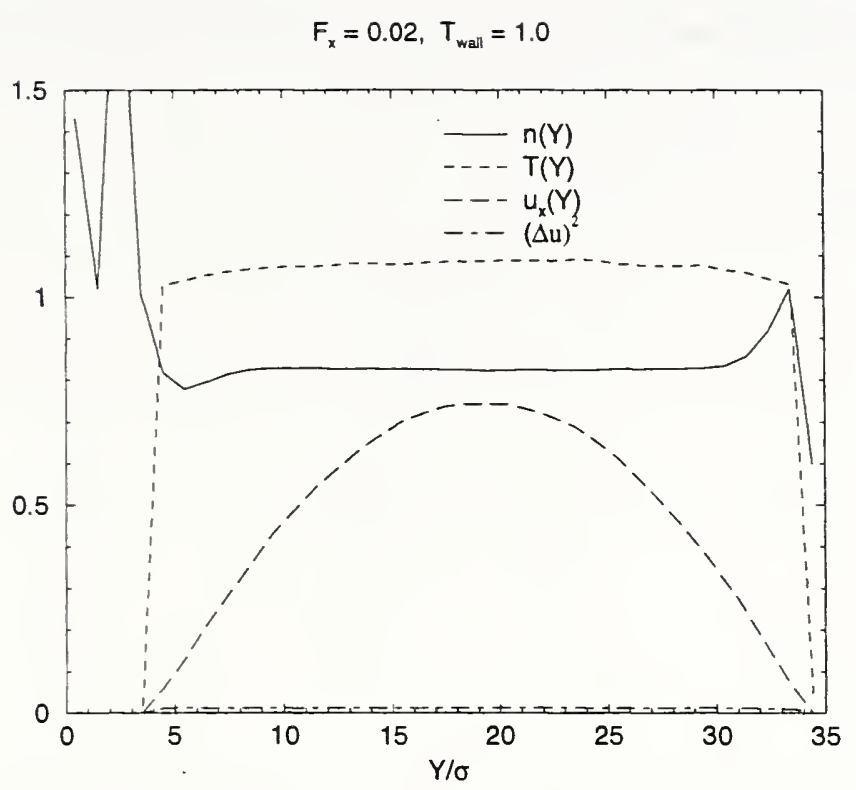

Fig. 2.3. The profiles for the density, temperature, flow velocity and mean square variation of the flow velocity are shown for the case with $F_{x}=0.02$

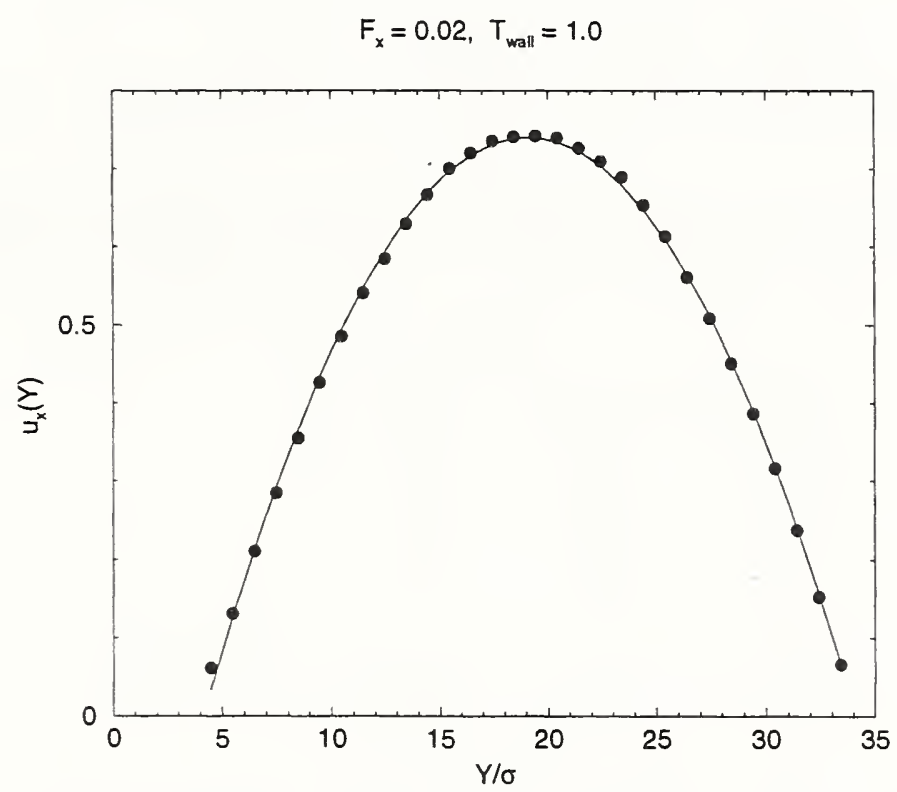

Fig. 2.4. The velocity profile, $u_{x}(Y)$, is shown for the case with $F_{x}=0.02$. The filled circles are the calculated values and the solid curve is the fitted profile, $u_{x}(Y)=A+B Y+c Y^{2}$.

as indicated in Fig. 2.5.

The transport coefficients are then determined using

$$
\eta=\frac{-n F_{x}}{2 u_{2}}
$$

and

$$
\lambda=\frac{\left(n F_{x}\right)^{2}}{12 \eta T_{4}}
$$




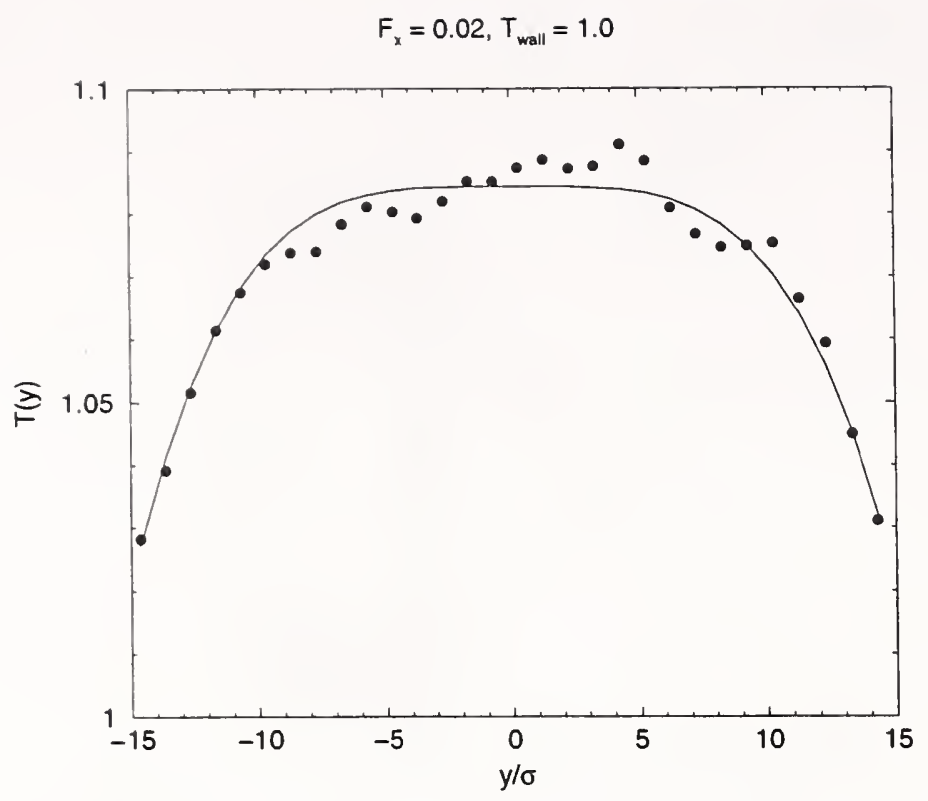

Fig. 2.5. The temperature profile, $T(y)$, is shown for the case with $F_{x}=0.02$. The filled circles are the calculated values and the solid curve is the fitted profile, $T(y)=T_{0}-T_{4} y^{4}$.

The results for several different values of the driving force $F_{x}$ are listed in Table 2 .

Table 2. The driving force $F_{x}$, and the computed values for the shear viscosity, $\eta$ and the thermal conductivity, $\lambda$ are listed for the case with the density $n=0.825$ and the wall temperature, $T_{\text {wall }}=1.0$.

\begin{tabular}{|c|c|c|}
\hline$F_{x}$ & $\eta$ & $\lambda$ \\
\hline 0.010 & 2.57 & 7.39 \\
0.015 & 2.49 & 7.34 \\
0.020 & 2.50 & 7.27 \\
0.025 & 2.47 & 7.44 \\
0.030 & 2.42 & 7.62 \\
0.040 & 2.35 & 8.00 \\
0.050 & 2.32 & 7.60 \\
\hline
\end{tabular}

These estimates for $\eta$ and $\lambda$ are consistent with published values of these transport coefficients obtained using the time correlation method of statistical mechanics $[4,7]$.

In order to get some idea for the uncertainties associated with these estimates for $\eta$ and $\lambda$, a set of ten runs of $1000 \tau$ duration were made for a driving force, $F_{x}=0.025$, for the same state as identified in Table 2. The viscosity values ranged from 2.43 to 2.49 with an average and uncertainty (the variance) of $2.45 \pm 0.018$. The themal conductivity values ranged from 7.11 to 8.32 with an an average and variance of $7.46 \pm 0.36$.

In order to obtain some idea of how long a simulation should run, the state identified in Table 2 was run for $200 \tau$. The cumulative time averages for $\eta, \lambda$, and $\mathrm{n}$ are shown in 
Fig. 2-6. The convergence for $\eta$ is quite good while the convergence for $\lambda$ is not convincing for $1000 \tau$.

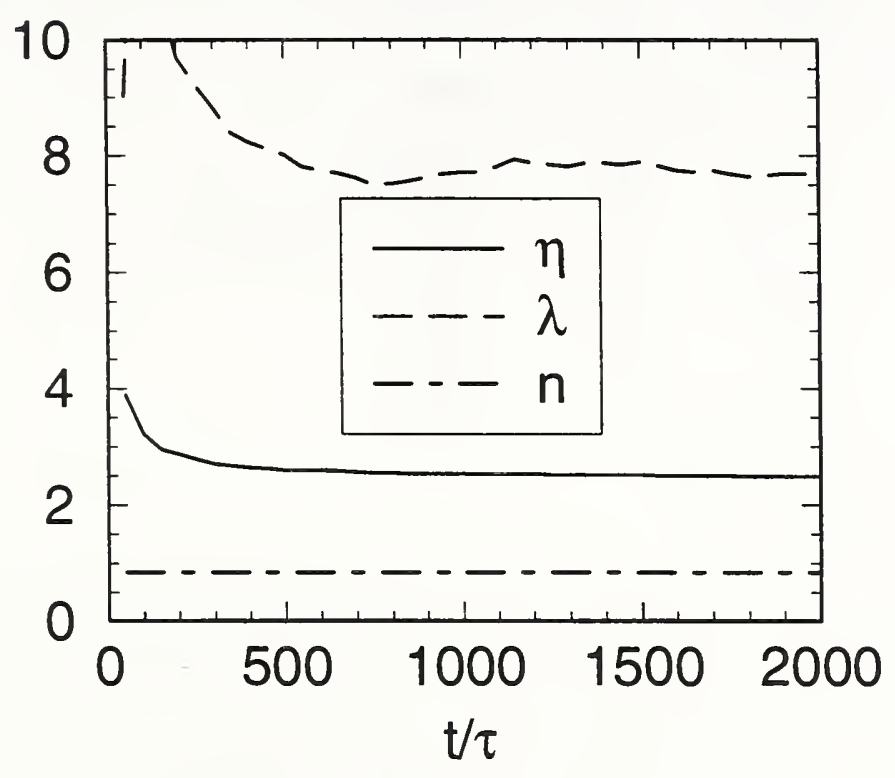

Fig. 2.6. The cumulative values for $\eta, \lambda$, and the number density $\mathrm{n}$ are shown a simulation of $2000 \tau$. The wall temperature is $\mathrm{T}_{\text {wall }}=1$, and $F_{x}=0.025$ The degree of convergence for $\eta$ is very good, while that for $\lambda$ is not as definite.

Some further tests were made by generating time averages of $10 \tau$ over an interval of $5000 \tau$. The shear viscosity values were closely grouped around the average while the thermal conductivity values were broadly distributed over the interval from 5 to 12 . From this we conclude that this method can generate the shear viscosity from a $1000 \tau$ run while much longer runs are needed to produce thermal conductivity estimates with reasonably small uncertainties. This is consistent with the observation that long runs are needed to reduce statistical scatter when the kinetic definition of local temperature is used.

In order to reduce the scatter in the thermal conductivity values, another set of ten simulations was performed with the local kinetic temperature being determined using the accumulated flow profile, $\mathbf{u}(\mathrm{y})$, rather than the instanteneous value. The duration of each of these simulations was $2000 \tau$ as this was found to be necessary for approximate convergence of the thermal conductivity. The averages and variances for the ten runs are $\eta=2.38 \pm 0.01, \lambda=7.34 \pm 0.40$, and $\mathrm{n}=0.832 \pm 0.00$. 
Table 3. The results for the shear viscosity, the thermal conductivity and the number density are listed for the ten simulations where the cumulative flow velocity was used to determine the kinetic temperature.

\begin{tabular}{|c|c|c|}
\hline$\eta$ & $\lambda$ & $\mathrm{n}$ \\
\hline 2.35 & 7.09 & 0.832 \\
2.36 & 7.39 & 0.832 \\
2.38 & 7.50 & 0.832 \\
2.39 & 7.70 & 0.832 \\
2.39 & 7.25 & 0.832 \\
2.40 & 8.19 & 0.832 \\
2.42 & 6.76 & 0.832 \\
2.35 & 7.33 & 0.832 \\
2.37 & 7.39 & 0.832 \\
2.38 & 6.76 & 0.832 \\
\hline
\end{tabular}

Alternative boundary regions.

Two variants of the boundary region were tested. The approach of Evans is rather inflexible in that the wall atoms are tethered to specific sites. There is some ambiguity about where the fluid meets the wall. Also, the process of changing the density of the fluid by changing the dimensions of the simulation cell involves some additional work. For these reasons, some alternatives were considered.

Suppose the regions at the ends of the cell are considered to be the wall region, as shown in Fig. 2.2. Molecules in the wall region are fluid molecules and are free to move in and out of the wall region. If a molecule reaches either end of the cell, it is diffusely reflected back into the fluid with momenta selected from a Boltzmann distribution with the wall temperature imposed. This proved to be rather inefficient in establishing a good temperature profile and was discontinued.

Instead, the approach that has been selected treats the two bins at the ends of the cell as the "wall". Periodic boundary conditions are imposed in all directions. At each time step, the molecules in the wall region are thermostated to the wall temperature by rescaling the momenta, and a zero net flow condition in the direction of the applied force that is imposed on the molecules. In other respects, this approach is the same as the other methods. This leads to coarse grained temperature and flow profiles that consistent are with those predicted by linear hydrodynamics. The shear viscosity and thermal conductivity are estimated by fitting quadratic and quartic profiles.

An extensive set of runs to implement this method has been made for a Lennard-Jones fluid with a mean density of 0.85 and average temperatures ranging from 0.9 to 2.75. The results are displayed in Fig. 2.7 for systems that were stabilized for $2000 \tau$ followed by a 
production run of an additional $2000 \tau$. The viscosities are in reasonable agreement with those listed in Table 1. For temperatures greater than 2, the thermal conductivities are larger than those in Table 1. Also, the scatter in the thermal conductivity values is large.

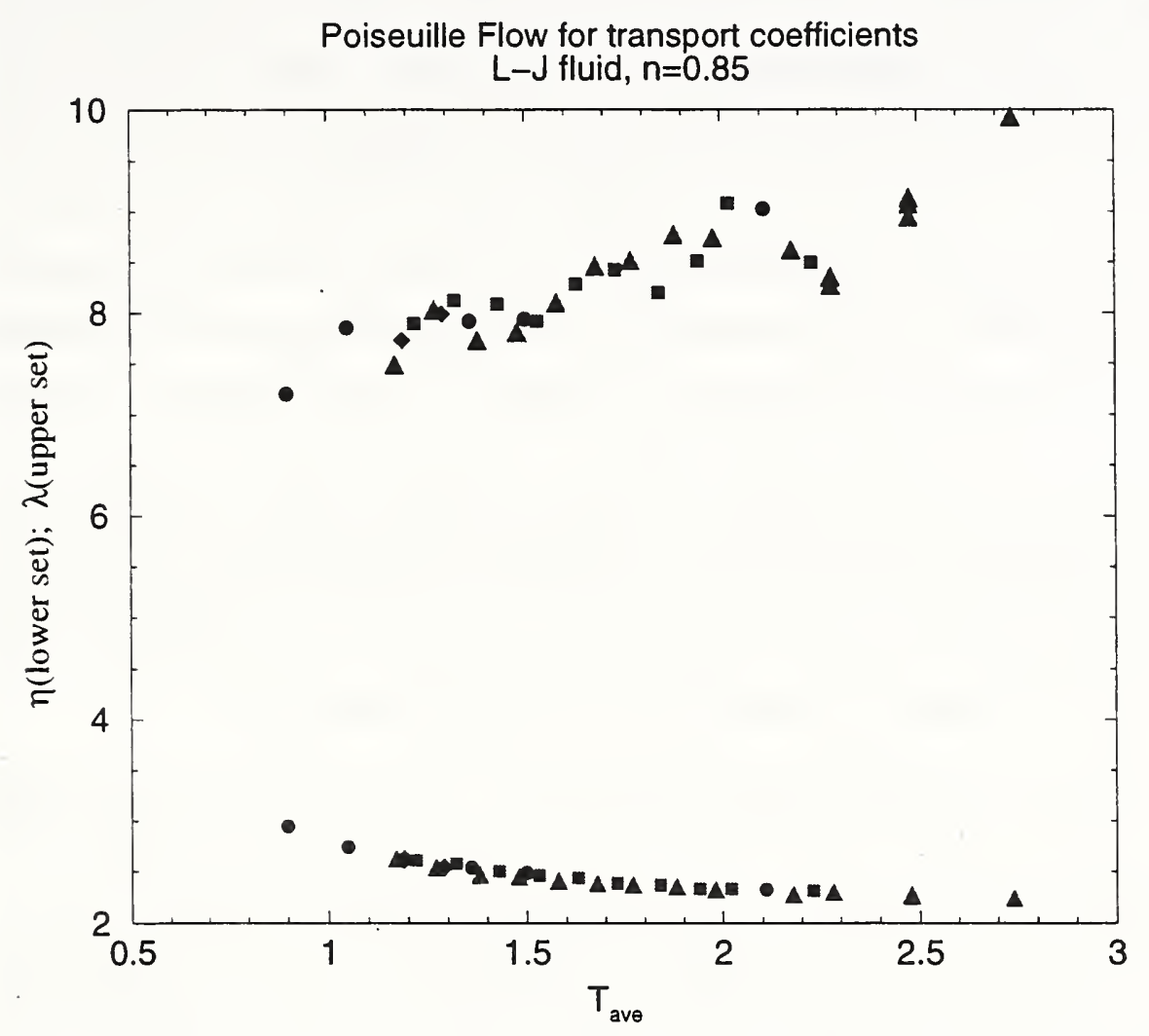

Fig. 2.7. The shear viscosity and thermal conductivity for a range of states for the Lennard-Jones fluid are shown here. The circles are for $\mathrm{F}_{x}=0.3$, the other symbols are for $\mathrm{F}_{x}=0.4$. Varying the number of layers in the system from 19 to 35 has minimal effect on the results.

\section{Momentum Inpulse Relaxation}

This approach to the determination of the shear viscosity uses the decay in time of a carefully designed transient momentum gradient and extracts the shear viscosity by matching the simulated momentum gradient to the hydrodynamic solution for the same problem [29]. The essence of the approach is the following.

Suppose the flow velocity is $u_{x}(y, t)$ given that the initial value is $u_{x}(y, 0)=a_{0} \exp \left(-b_{0} y^{2}\right)$. If there is no pressure gradient or external force present, the flow velocity satisfies

$$
\frac{\partial u_{x}(y, t)}{\partial t}=\nu \frac{\partial^{2} u_{x}(y, t)}{\partial y^{2}}
$$

The solution is

$$
u_{x}(y, t)=\left[\frac{a_{0}}{\left(1+t / t_{0}\right)}\right]^{1 / 2} \exp \left[-b_{0} y^{2} /\left(1+t / t_{0}\right)\right]
$$


where $t_{0}=1 /\left(4 \nu b_{0}\right)$, and $\nu$ is the kinematic viscosity, the shear viscosity divided by the density.

While this appears to be a straightforward simulation, there is a problem with the periodic boundary conditions in the $y$-direction. If the usual condition of not changing the $y$ component of the momentum when a molecule leaves the simulation cell through a plane perpendicular to the $y$-axis and its periodic image enters the cell through the opposite side of the cell, the flow velocity will not decay to zero at long times. To take care of this, a special procedure is introduced to permit the flow velocity to decay as if the system were infinite. This is done by requiring the $x$-component of the velocity of the particle entering the simulation cell to be consistent with the current estimate of the Gaussian velocity profile.

The process of determining the Gaussian profile at a time $t$ is achieved by partitionaing the simulation cell into bins parallel to the $x-z$ plane. The flow velocity of each bin is just the average $x$-component of the velocity of the particles in each bin at that time. The resulting velocity profile is then fitted to a two parameter Gaussian function,

$$
u_{x}(t)=u_{p}(t) \exp \left(-y^{2} / \sigma^{2}(t)\right)
$$

The fit parameters, $u_{p}(t)$ and $\sigma^{2}(t)$, are then used to determine by extrapolation the $x$ component of the velocity of the image molecule, $u_{x}^{E}(t)$, just before it enters the simulation cell as

$$
u_{x}^{E}(t)=u_{p}(t) \exp \left(-y^{2}(t-\Delta t) / \sigma^{2}(t)\right)
$$

where $y(t-\Delta t)$ is the $y$-coordinate of the image molecule at the time step before it enters the cell. The decay of the imposed flow velocity is realized by assigning $u_{x}^{E}(t)$ to the $x$-component of the velocity of the particle entering the cell.

This fitting procedure is also used to determine the shear viscosity. Both $u_{p}(t)$ and $\sigma^{2}(t)$ can be used to estimate the characteristic time $t_{0}$ and therefore the viscosity. The peak velocity, $u_{p}(t)$ has the form

$$
\frac{A}{(1+B t)^{1 / 2}}
$$

so that

$$
\eta=\frac{\rho B}{4 b_{0}} .
$$

Also, the variance $\sigma^{2}(t)$ is a linear function of time and may be used to provide another estimate of $t_{0}$.

In the reported tests, the cell had dimensions $\mathrm{L} / 2 \times \mathrm{L} \times \mathrm{L} / 2$ and 19 bins were used. The dimensions were such that on the order of 100 molecules were in each bin. The simulations 
were performed by equilibrating the fluid without any imposed flow field, and then running the system with the initially imposed flow for about $10 \mathrm{ps}$. This sequence was repeated 20 times with separate initial conditions. Results are available for the Lennard-Jones fluid and for "butane". The results for the shear viscosity compare favorably with those obtained both with Green-Kubo time correlations and with nonequilibrium applied shear simulations. The required run times for this method are at least and order of magnitude smaller that for the other methods.

Some possible concerns are about the uniformity of the density (not discussed) and about appropriate parameters for the imposed initial conditions (extensively discussed below). There has not been any proposal for a similar approach to determining the thermal conductivity of a fluid, although the decay of a temperature pulse has been used in a molecular dynamics simulation to estimate the thermal conductivity of a superlattice crystal [30]. It would require a careful analysis of thermal expansion effects to implement such an approach for a liquid.

\section{Implementation}

The Momentum Impulse Relaxation scheme has been implemented as follows. First, a set of coordinates for 2000 molecules at the desired temperature and density are generated with a uniform momentum distribution. Then a momentum pulse with amplitude $a(0)$ and width $b(0)$ are imposed and the system evolves for $10 \tau$. The evolution of $a(t)$ and $b(t)$ are determined using 19 bins uniformly distributed in the simulation cell. This sequence is repeated 20 times and the average of $a(t)$ is used to determine $\eta$ using eq. 3.5. Some results for $\mathrm{n}=0.85$ and three values of $a(0)(0.3,0.4$, and 0.5$)$ and $b(0)=0.25$ are shown in Fig. 3.1. If $a(0)$ were smaller than 0.3 , it was difficult to extract a useful signal. These values for the viscosity are consistent with those listed in Table 1.

While it is straightforward to set up the momentum pulse in an initially "equilibrium" fluid state, I have encountered serious difficulties in realizing results in the form of eq. 3.2 over extended times. The task is to determine the amplitude and width of the momentum pulse by fitting the calculated profile to a Gaussian form. This is a nonlinear fitting task, but since the changes from one time step to the next are small, this is not a difficult thing to do using a steepest descent method. For relatively short times, the fitted amplitude and width conform the the scaled forms. However, for times longer than one time unit or so, there are serious, systematic departures from the scaled form.

My conjecture is that the suggested way of dealing the the "leakage" of momentum out of the simulation cell is not adequate. Actually only a few particles cross the cell boundary at any one time so the flux out of the simulation cell is small. Since this method, while requiring relatively short overall simulation times is potentially useful, it is not at this time 


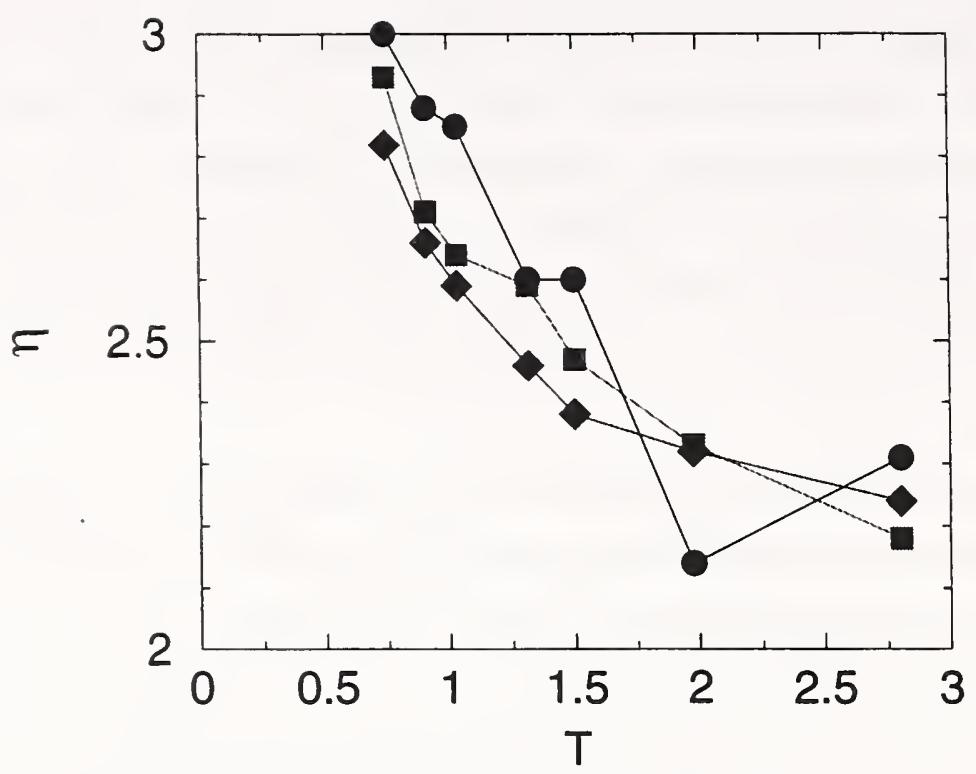

Fig. 3.1. The estimated values of the shear viscosity and indicated for three values of the initial amplitude of the momentum pulse. The circles are for $a(0)=0.3$, squares are for $a(0)=0.4$, and the diamonds are for $a(0)=0.5$. The lines connecting the points are to guide the eye.

sufficiently robust to warrant further investigation here.

\section{Reversed perturbation nonequilibrium MD}

Another approach to obtaining transport coefficients from molecular simulations is based on the following observations. A transport coefficient is the proportionality coefficient between a current and a force. For example,

$$
\mathbf{J}=-\lambda \frac{d T}{d z}
$$

where $\lambda$ is the thermal conductivity, $d T / d z$ is the temperature gradient that drives the current, and $\mathbf{J}$ is the heat current. In the "usual" molecular dynamics approaches to determining a transport coefficient, the driving force is specified and the current is calculated. In the reversed perturbation approach, the current is established by some scheme and the resulting gradient is determined [31, 32, 33, 34]. In these papers, the methodology for determining thermal conductivity and shear viscosity are examined. Some initial applications for the determination of the thermal conductivity for a variety of systems have been reported $[35,36,37,38,39,40,41]$. Extensions of the method to diffusion in mixtures has also been described $[42,43]$.

The essential feature of this approach is to generate a known current that is stationary in time. This is done by separating the simulation cell of dimensions $L_{x} \times L_{y} \times L_{z}$ into layers as indicated in Fig. 4.1. The central layer and the layer at one end of the cell are special. At some interval $\tau_{\text {trans }}$, a property of a molecule in each of these layers is interchanged 
such that energy and momentum are conserved and that a gradient between the layers is established. Because of periodic boundary conditions in all three dimensions, a current flows from the central layer to the layer at one end and also to the periodic image of the end layer. Once a stationary current is established, one then determines the corresponding gradient by sampling the temperature or flow velocity in each of the layers.

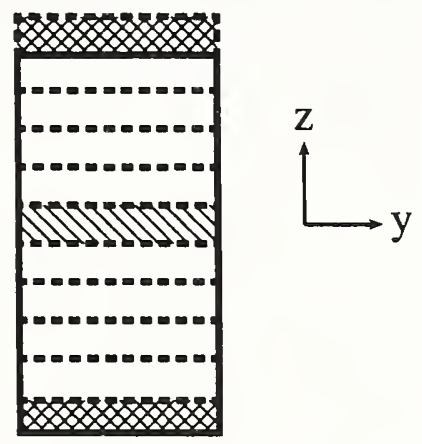

Fig. 4.1. A sketch of the simulation scheme. The dark frame indicates the simulation cell (the $\mathrm{x}$-direction is normal to the page). The dashed lines indicate the boundaries of the layers. The cross hatched layers are the end layer and its periodic image and the tilted line layer is the central layer. Note that the total number of layers is even so the the distance between the "walls" and the central cell is the same in both directions.

First let us consider the way a heat current is established. Let the central layer be the hot one and the other layer be the cold one. At time intervals of $\tau_{\text {trans }}$, the velocity vectors of the molecule with the largest kinetic energy of molecules in the cold layer and the molecule with the lowest kinetic energy in the hot layer are exchanged. This conserves the total momentum and energy of the fluid and eventually results in a temperature gradient between the central layer and the two crosshatched layers shown in Fig. 3.1. The heat current is then the difference in the kinetic energy transfered between the hot and cold layers averaged over time and over the area of the layer. If $v_{h}^{2}$ and $v_{c}^{2}$ are the squares of the velocities of the hot and cold molecules, then the time averaged heat current, $J$, is

$$
J=\frac{\sum_{\text {transfers }} m\left(v_{h}^{2}-v_{c}^{2}\right) / 2}{2 t L_{x} L_{y}}
$$

where $t$ is the averaging time and transfers is the set of energy transfers. If an exchange 
of kinetic energy occurs once every $\tau_{\text {trans }}$ then $t / \tau_{\text {trans }}$ is the number of transfer events.

The temperature gradient is obtained from the temperature profile of the layers. The temperature of layer $\mathrm{k}, T_{k}$, is $2 / 3$ times the average kinetic energy of the $N_{k}$ molecules in layer $\mathrm{k}$ divided by Boltzmann's constant $k_{B}$.

$$
T_{k}=\frac{\sum_{i \in k}^{N_{k}} m_{i} v_{i}^{2}}{3 N_{k} k_{B}}
$$

Determining the average temperature of the layers is less time consuming that evaluating the time correlation function of the properly defined heat current that goes into the Green-Kubo expression for the thermal conductivity. The time consuming part is the establishment of a stationary temperature gradient.

A similar scheme is used to establish a momentum flux. Instead of picking the hottest and coldest molecules in the central and end layers, one picks the molecule with the largest $y$-component of velocity to the right in the central layer and the molecule with the largest $y$-component of velocity in the opposite direction in the end layer and interchanges the velocity vectors. Again the total momentum and energy are conserved. The result is a gradient of the $z$-direction of the $y$-component of the layer velocity. The layer velocity, $u_{k}$ is just the average velocity of the molecules in the $k$-th layer. The momentum transfer for a transfer event, $\Delta p_{y}$, is just the difference in the $y$-components of momentum of the selected molecules and the shear viscosity, $\eta$, is then

$$
\eta=-\frac{\sum_{\text {transfer }} \Delta p_{y}}{2 t L_{x} L_{y} d u / d z}
$$

and the shear gradient, $d u / d z$, is the slope of the $u_{y}(k)$ vs $z(k)$ curve.

Some limits on the applicability of this approach have been noted $[44,40]$. If the switching time $\tau_{\text {trans }}$ is too long, then the gradients do not develop as intended and the simple connection between the flux and the temperature or velocity profile is lost.

\section{Implementation}

This approach requires a separate simulation for the determination of the shear viscosity and the thermal conductivity. First, we examine the results for the thermal conductivity, $\lambda$, for the state with $\mathrm{T}=1.0$ and the density $\mathrm{n}=0.887$. The results for a set of ten (10) sequential simulations of duration $1000 \tau$ ( $10^{5}$ time steps) are listed in Table 4 . The temperature profile for one of these runs is displayed in Fig. 4.2 to illustrate the sort of profiles involved in determining $\mathrm{dT}(\mathrm{y}) / \mathrm{dy}$.

The corresponding results for the shear viscosity are found in Fig. 4.3 and Table 5. 
A separate pair of runs, one each for $\eta$ and for $\lambda$, of $10^{6} \Delta t$ were also made. the results are: $\Delta P=0.0598, \eta=3.75$ and $3.76 ; J_{Q}=0.0737, \lambda=8.76$ and 8.79 . The results of the longer runs are consistent with those listed in Tables 4 and 5 .

As a further check on this method, a series of runs were made for the states listed in Table 1.1. The results for $\mathrm{n}=0.85$ are displayed in Fig. 4.4.

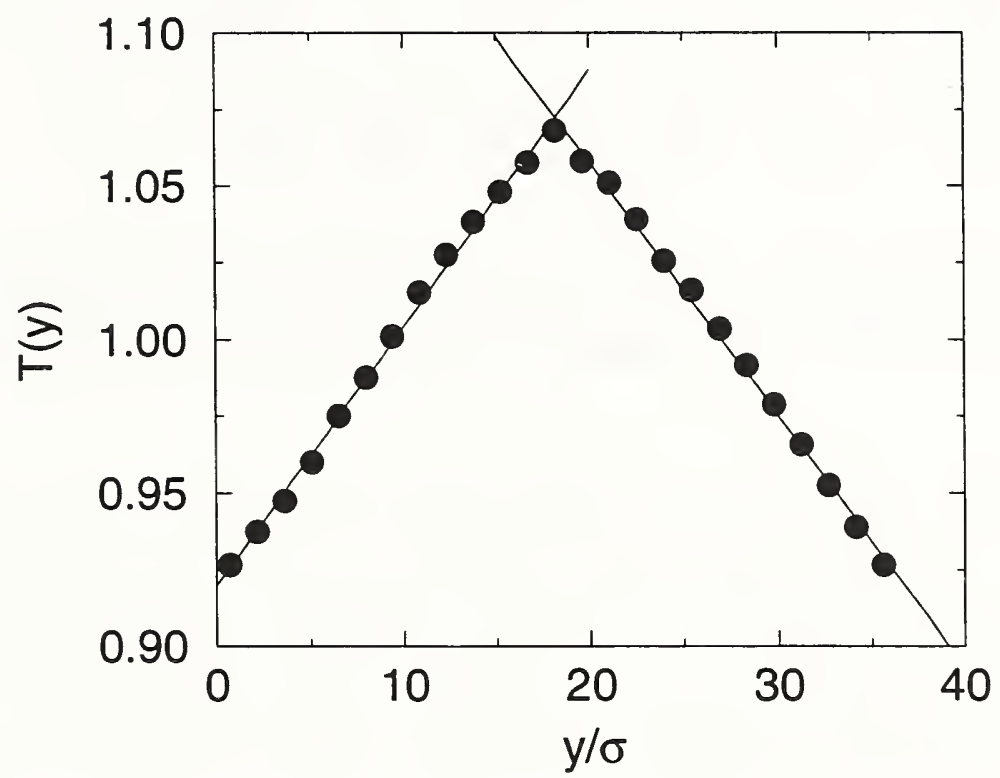

Fig. 4.2. The temperature profile, $T(y)$, is shown for a case with switching interval $=50 \Delta t$. The solid circles are the computed profile and the solid lines are the least-squares fit to the profile.

Table 4. The results for the thermal conductivity are listed for 10 runs, each of $1000 \tau$ duration; $J_{Q}=0.0735 \pm 0.004$ and $\lambda=8.53 \pm 0.26$. The two values of the thermal conductivity listed for each flux are determined from the two parts of the profile shown in Fig. 4.2. The switching interval is $50 \Delta t$.

\begin{tabular}{|c|c|c|}
\hline$J_{Q}$ & $\lambda$ & $\lambda$ \\
\hline 0.0737 & 8.40 & 8.44 \\
0.0732 & 8.05 & 8.16 \\
0.0738 & 8.99 & 8.97 \\
0.0741 & 8.56 & 8.54 \\
0.0735 & 8.54 & 8.60 \\
0.0730 & 8.34 & 8.20 \\
0.0740 & 8.78 & 8.69 \\
0.0730 & 8.35 & 8.38 \\
0.0731 & 8.47 & 8.44 \\
0.0738 & 8.80 & 8.97 \\
\hline
\end{tabular}




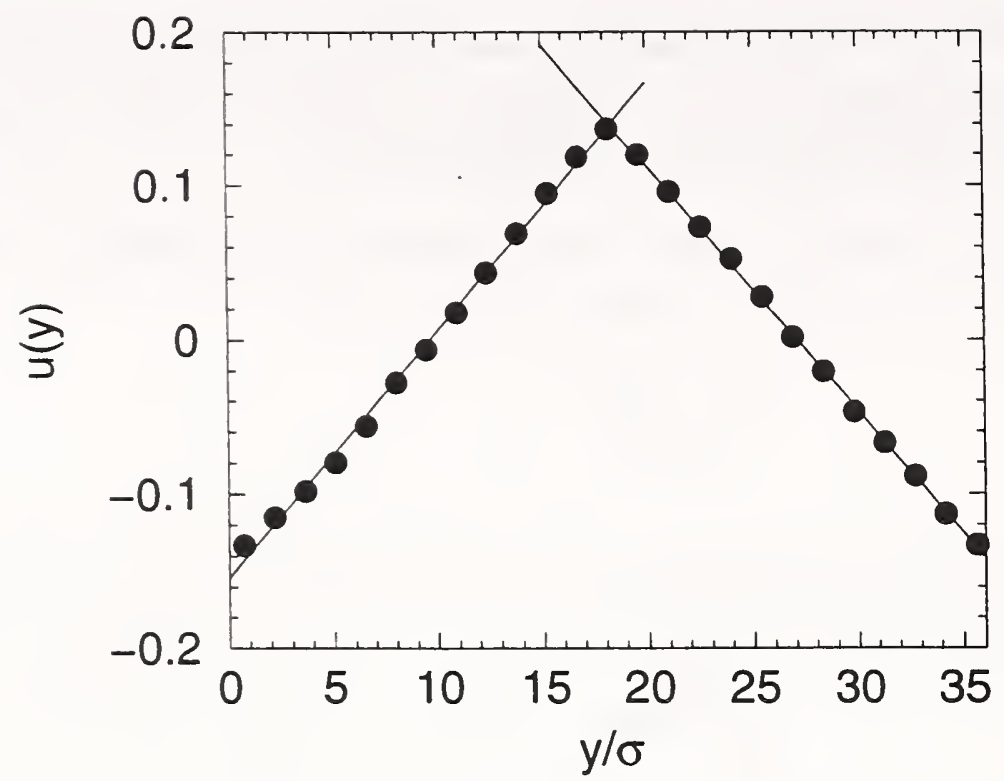

Fig. 4.3. The momentum profile, $u(y)$, is shown for a case with switching interval $=50 \Delta t$. The solid circles are the computed profile and the solid lines are the least-squares fit to the profile.

Table 5. The results for the shear viscosity are listed for 10 separate runs, each of $1000 \tau$ duration; $\Delta \mathrm{P}=0.0599 \pm 0.0002$ and $\eta_{S}=3.80 \pm 0.13$. The two values of the shear viscosity listed for each flux are determined from the two parts of the profile shown in Fig. 4.3. The switching interval is $\tau_{\text {trans }}=50 \Delta t$.

\begin{tabular}{|c|c|c|}
\hline$\Delta P$ & $\eta$ & $\eta$ \\
\hline 0.0601 & 4.05 & 4.01 \\
0.0598 & 3.88 & 3.95 \\
0.0596 & 3.55 & 3.61 \\
0.0599 & 3.76 & 3.66 \\
0.0598 & 3.85 & 3.79 \\
0.0601 & 3.78 & 3.87 \\
0.0600 & 3.93 & 3.90 \\
0.0599 & 3.70 & 3.69 \\
0.0601 & 3.85 & 3.74 \\
0.0598 & 3.72 & 3.78 \\
\hline
\end{tabular}

The interval between swap events, $\tau_{\text {trans }}$ is an important parameter that determines the magnitude of the temperature or momentum gradient. Some tests of the sensitivity of the results have been made for the $\mathrm{T}=3.564, \mathrm{n}=0.65$ state of Table 1 . The results, that were generated by Jim Rainwater [45], are listed in Table 6. These results indicate that $0.25 \tau<\tau_{\text {transfer }}<1.0 \tau$ is an acceptable range of switching times. The Green-Kubo estimates for this state are $\lambda=5.4 \pm 0.3$ and $\eta_{S}=1.15 \pm 0.03$. 


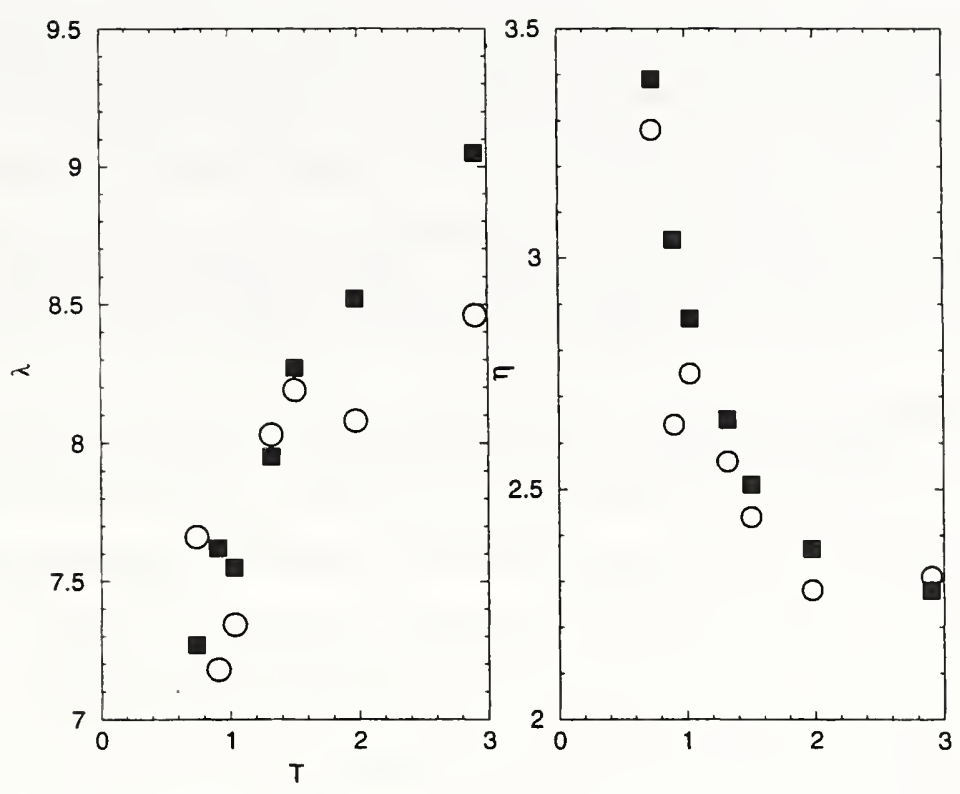

Fig. 4.4. The results of this method (black squares) are compared with the Green-Kubo results (open circles) of Table 1.1 for the $\mathrm{n}=0.85$ states.

Table 6. The results for the shear viscosity and thermal conductivity as a function of the switching time $\tau_{\text {trans }}$ are listed for the $\mathrm{T}=3.564, \mathrm{n}=0.65$ state of Table 1 .

\begin{tabular}{|c|c|c|}
\hline$\tau_{\text {trans }} / \tau$ & $\lambda$ & $\eta$ \\
\hline 0.06 & 5.06 & \\
0.12 & 5.12 & 1.13 \\
0.25 & 5.20 & 1.16 \\
0.40 & 5.19 & \\
0.50 & 5.17 & 1.16 \\
1.00 & 5.21 & 1.19 \\
2.00 & 5.11 & 1.22 \\
4.00 & 5.11 & 1.21 \\
\hline
\end{tabular}

\section{Discussion}

First, we consider the relative wall clock/CPU time needed to obtain "satisfactory" estimates for the shear viscosity and the thermal conductivity using the various methods examined. For purposes of reference, we note that a Green-Kubo simulation of duration $10^{4} \tau$ generates estimates with approximately $5 \%$ uncertainty in the shear viscosity and about $10 \%$ uncertainty in the thermal conductivity.

As indicated by the values in Table 3 and Figure 2.6, the results for Poiseuille flow are that a simulation of $10^{3} \tau$ will produce shear viscosity estimates with an uncertainty of less than 5\%. Even allowing for the larger system size (a factor of 5), this requires less CPU time than the Green-Kubo method. That is not the case for the thermal conductivity as a simulation nearly 20 times longer is needed to achieve a $10 \%$ uncertainty level. 
For the Reversed Pertubation Nonequilibrium Molecular Dynamics method, the results in Tables 4 and 5 suggest that the $5 \%$ uncertainty for the shear viscosity and $10 \%$ uncertainty for the thermal conductivity can be obtained with $10^{3} \tau$ simulations. Now allowing for the need to establish the gradients and the larger system size involved, the individual transport coefficients can be obtained in a time comparable to that required for the Green-Kubo method. If both coefficients are needed, the Green-Kubo method would be a bit faster.

The Momentum Impulse Relaxation method is considerably faster than any of the other methods. However, until the problem of departures from the scaling of the solutions from the hydrodynamic predictions are resolved, this method remains a research topic.

There are some "loose ends" with the nonequilibrium simulation methods. These are the "optimal" switching rate for the Reversed Perturbation method and the appropriate magnitude of the driving force for Poiseuille flow. Another, potentially more significant factor is the minimum system size needed to obtain proper profiles. The simulations discussed here contained over 2000 atoms. If this could be reduced to 1000, the advantages of the nonequilibrium methods over the Green-Kubo method would be significant.

Another factor to be considered concerns problems associated with these methods when applied to molecular fluids as opposed to the monatomic Lennard-Jones fluid. If we restrict our attention to rigid molecules, then there do not appear to be serious problems provided the momenta and forces are center-of-mass values and molecular currents are used [46, 47]. Further discussion of this topic lies outside the scope of this report.

Finally, we note that cases where the viscosity of the fluid is sufficiently large that viscoelastic effects are present have not been examined. For those situations, it is known that the correlation times for the shear viscosity correlation functions appearing in eq. 1.4 become much longer than occurs for any of the cases examined here. In that situation, the Green-Kubo method will require much longer simulations in order to obtain statistically independent samples. It is not known how the other methods will behave for such systems.

Acknowledgement: This study was supported by an Exploratory Research Award from the Chemical Science and Technology Laboratory of NIST.

\section{References}

[1] J. P. Hansen and I. R. McDonald, Theory of Simple Liquids, 253-363 (Academic Press, New York, 1986).

[2] P. Schofield, Computer simulation studies of the liquid state, Comput. Phys. Comm. 5, 17-23 (1973).

[3] M. P. Allen and D. J. Tildesley, Computer Simulation of Liquids, 145-152 (Clarendon Press, Oxford, 1987). 
[4] R. Vogelsang, C. Hoheisel, and M. Lukas, Shear viscosity and thermal conductivity of the Lennard-Jones liquid computed using molecular dynamics and predicted by a memory-function model for a large number of states, Mol. Phys. 64, 1203-1213 (1988).

[5] D. M. Heyes, Transport coefficients of Lennard-Jones fluids: A molecular-dynamics and effective-hard-sphere treatment, Phys. Rev. B 37, 5677-5696 (1988).

[6] D. M. Heyes and J. G. Powles, Information theory applied to the transport coefficients of Lennard-Jones fluids, Mol. Phys. 71, 781-800 (1990).

[7] R. L. Rowley and M. M. Painter, Diffusion and viscosity equations of state for a Lennard-Jones fluid obtained from molecular dynamics simulations, Int. J. Thermophys. 18, 1109-1121 (1997).

[8] K. Meier, A. Laesecke, and S. Kabelac, A Molecular Dynamics Simulation Study of the Self-Diffusion Coefficient and Viscosity of the Lennard-Jones Fluid, Int. J Thermophysics 22, 161-173 (2002).

[9] K. Meier, A. Laesecke, and S. Kabelac, Transport coefficients of the Lennard-Jones model fluid. I. Viscosity, J. Chem. Phys. 121, 3671-3687 (2004).

[10] G. A. Fernández, J. Vrabec, and H. Hasse, A molecular simulation study of shear and bulk viscosity and thermal conductivity of simple real fluids, Fluid Phase Equilibria 221, 1157-163 (2004).

[11] M. S. Green, Markov random processes and the statistical mechanics of time-dependent phenomena. II. Irreversible processes in fluids, J. Chem. Phys. 22, 398-413 (1954).

[12] M. S. Green, Comment on a paper of Mori on time-correlation expressions for transport properties, Phys. Rev. 119, 829-8330 (1960).

[13] B. L. Holian and D. J. Evans, Shear viscosities away from the melting line. A comparison of equilibrium and nonequilibrium molecular dynamics, J. Chem. Phys. 78, 5147-5150 (1983).

[14] J. J. Erpenbeck, Shear viscosity of the Lennard-Jones fluid near the triple point: GreenKubo results, Phys. Rev. A 38, 6244-6266 (1988).

[15] R. Vogelsang, C. Hoheisel, and G. Ciccotti, Thermal conductivity of the Lennard-Jones liquid by molecular dynamics calculations, J. Chem. Phys. 86, 6371-6375 (1987).

[16] I.-C. Yeh and G. Hummer, System-size dependence of diffusion coefficients and viscosities from molecular dynamics simulations with periodic boundary conditions, J. Phys. Chem. B 108, 15873-15879 (2004). 
[17] P. E. Smith and W. F. van Gunsteren, The viscosity of $S P C$ and $S P C / E$ water at 277 and $300 \mathrm{~K}$, Chem. Phys. Lett. 215, 315-318 (1993).

[18] B. D. Todd and D. J. Evans, Temperature profile for Poiseuille flow, Phys. Rev. E 55, 2800-2807 (1997).

[19] B. D. Todd and D. J. Evans, The heat flux vector for highly inhomogeneous nonequilibrium fluids in very narrow pores, J. Chem. Phys. 103, 9804-9809 (1995).

[20] P. J. Daivis, K. P. Travis, and B. D. Todd, A technique for the calculation of mass, energy, and momentum densities at planes in molecular dynamics simulations, J. Chem. Phys. 104, 9651-9653 (1996).

[21] K. P. Travis, B. D. Todd, and D. J. Evans, Departure from Navier-Stokes hydrodynamics in confined liquids, Phys. Rev. E 55, 4288-4295 (1997).

[22] J. Delhommelle and D. J. Evans, Configurational temperature profile in confined fluids. I. Atomic fluid, J. Chem. Phys. 114, 6229-6235 (2001).

[23] J. Delhommelle and D. J. Evans, Poiseuille flow of a micropolar fluid, Mol. Phys. 100, 2857-2865 (2002).

[24] O. G. Jepps, G. Ayton, and D. J. Evans, Microscopic expressions for the thermodynamic temperature, Phys. Rev. E 62, 4757-4763 (2000).

[25] I. Yeh and M. L. Berkowitz, Ewald summation for systems with slab geometry, J. Chem. Phys. 111, 3155-3162 (1999).

[26] J. Lekner, Summation of Coulomb fields in computer-simulated disordered systems, Physica A 176, 485-498 (1991).

[27] R. D. Mountain, Molecular dynamics study of thin water-acetonitrile films, J. Phys. Chem. B 105, 6556-6561 (2001), References to earlier work on the Lekner scheme are included in this paper.

[28] G. J. Martyna, M. L. Klein, and M. Tuckerman, Nosé-Hoover 'chains: The canonical ensemble via continuous dynamics, J. Chem. Phys. 97, 2635-2643 (1992).

[29] G. Arya, E. J. Maginn, and H. Chang, Efficient viscosity estimation from molecular dynamics simulation via momentum inpulse relaxation, J. Chem. Phys. 113, 2079$2087(2000)$.

[30] B. C. Daly, H. J. Maris, K. Imamura, and S. Tamura, Molecular dynamics calculation of the thermal conductivity of superlattices, Phys. Rev. B 60, 024301 (2002).

[31] F. Müller-Plathe, A simple nonequilibrium molecular dynamics method for calculating the thermal conductivity, J. Chem. Phys. 106, 6082-6085 (1997). 
[32] F. Müller-Plathe, Reversing the pertubation in nonequilibrium molecular dynamics: An easy way to calculate the shear viscosity of fluids, Phys. Rev. E 59, 4894-4898. (1999).

[33] P. Bordat and F. Müller-Plathe, The shear viscosity of molecular fluids: A calculation by reverse nonequilibrium molecular dynamics, J. Chem. Phys. 116, 3362-3369 (2002).

[34] C. Nieto-Draghi and J. B. Avalos, Non-equilibrium momentum exchange algorithm for molecular dynamics simulation of heat flow in multicomponent systems, Mol. Phys. 101, 2303-2307 (2003).

[35] B. Bresme, B. Hafskjold, and I. Wold, Nonequilibrium molecular dynamics study of heat conduction in ionic systems, J. Phys. Chem. 100, 1879-1888 (1996).

[36] D. Bedrov and G. D. Smith, Thermal conductivity of molecular fluids from molecular dynamics simulations: Application of a new imposed-flux method, J. Chem. Phys. 113, 8080-8084 (2000).

[37] D. Bedrov, G. D. Smith, and T. D. Sewell, Thermal conductivity of liquid octahydro1,3,5,7-tetranitro-1,3,5,7-tetrazocine (HMX) from molecular dynamics simulations, Chem. Phys. Letters 324, 64-68 (2000).

[38] F. Bresme, Equilibrium and nonequilibrium molecular-dynamics simulations of the central force model of water, J. Chem. Phys. 115, 7564-7574 (2001).

[39] C. Nieto-Draghi, J. B. Avalos, and B. Rousseau, Transport properties of diemethyl sulfoxide aqueous solutions, J. Chem. Phys. 119, 4782-4789 (2003).

[40] Z. Donkó and P. Hartmann, Thermal conductivity of strongly coupled Yukawa liquids, Phys. Rev. E 69, 0166405 (2004).

[41] C. W. Padgett and D. W. Brenner, Influence of chemisorption on the thermal conductivity of single-wall carbon nanotubes, Nano Letters 4, 1051-1053 (2004).

[42] D. Reith and F. Müller-Plathe, On the nature of thermal diffusion in binary LennardJones liquids, J. Chem Phys. 112, 2436-2443 (2000).

[43] P. Bordat, D. Reith, and F. Müller-Plathe, The influence of interaction details on the thermal diffusion in binary Lennard-Jones liquids, J. Chem. Phys. 115, 8978-8982 (2001).

[44] C. P. Calderon and W. T. Ashurst, Comment on "Reversing the perturbation in nonequilibrium molecular dynamics: An easy way to calculate the shear viscosity of liquids", Phys. Rev. E 66, 103201 (2002). 
[45] J. Rainwater, NIST Boulder Laboratory, Boulder, CO.

[46] D. J. Evans and S. Murad, Thermal conductivity in molecular fluids, Mol. Phys. 68, 12191223 (1989).

[47] S. T. Cui, P. T. Cummings, and H. D. Cochran, The calculation of the viscosity from the autocorrelation function using molecular and atomic stress tensors, Mol. Phys. 88, 1657-1664 (1996). 

